.

NASA Breakthrough Propulsion Physics Program

Marc G. Millis

Lewis Research Center, Cleveland, Ohio

Prepared for the

Second Symposium on Realistic Near-Term Advanced Scientific Space Missions cosponsored by the International Academy of Astronautics and Politecnico di Torino Aosta, Italy, June 29-July 1, 1998

National Aeronautics and

Space Administration

Lewis Research Center 
Available from

NASA Center for Aerospace Information 7121 Standard Drive

Hanover, MD 21076

Price Code: A03
National Technical Information Service 5287 Port Royal Road Springfield, VA 22100 Price Code: $\mathrm{A} 03$ 


\title{
NASA BREAKTHROUGH PROPULSION PHYSICS PROGRAM
}

\author{
MARC G. MILLIS ${ }^{1}$ \\ NASA Lewis Research Center \\ 21000 Brookpark Road, MS 60-4 \\ Cleveland, Ohio 44135, USA
}

\begin{abstract}
In 1996, NASA established the Breakthrough Propulsion Physics program to seek the ultimate breakthroughs in space transportation: propulsion that requires no propellant mass, propulsion that attains the maximum transit speeds physically possible, and breakthrough methods of energy production to power such devices. Topics of interest include experiments and theories regarding the coupling of gravity and electromagnetism, vacuum fluctuation energy, warp drives and wormholes, and superluminal quantum effects. Because these propulsion goals are presumably far from fruition, a special emphasis is to identify affordable, near-term, and credible research that could make measurable progress toward these propulsion goals. The methods of the program and the results of the 1997 workshop are presented. This Breakthrough Propulsion Physics program, managed by Lewis Research Center, is one part of a comprehensive, long range Advanced Space Transportation Plan managed by Marshall Space Flight Center.
\end{abstract}

\section{INTRODUCTION}

New theories and phenomena have emerged in recent scientific literature that have reawakened consideration that propulsion breakthroughs may be achievable - the kind of breakthroughs that could make human voyages to other star systems possible. This includes literature about warp drives, wormholes, quantum tunneling, vacuum fluctuation energy, and the coupling of gravity and electromagnetism. This emerging science, combined with the realization that rockets are fundamentally inadequate for interstellar exploration, led NASA to establish the "Breakthrough Propulsion Physics" program in 1996.

This paper introduces this program and several of the candidate research approaches that have already been identified. In particular, this paper explains the methods used by this program to conduct such visionary work as a lesson for other institutions who may also wish to begin similar programs. Also, to give an indication of some of the possible next research steps, the results of the 1997 workshop are presented.

\section{BACKGROUND}

Prior to 1996 the implications of emerging science to the challenges of space propulsion were only sporadically studied, and then mostly by individual researchers who did so on their own time. Occasionally research and workshops were formally supported [111], but progress was generally slow.

In 1996, the NASA Marshall Space Flight Center (MSFC) was tasked to formulate a comprehensive strategy for advancing propulsion for the next 25 years and they were requested to make this strategy more visionary than previous plans. This strategy, called the "Advanced Space Transportation Program (ASTP)," spans the nearer-term technology improvements all the way through seeking the breakthroughs that could revolutionize space travel and enable interstellar voyages [12].

To address the most visionary end of this scale, MSFC sought out the work of the NASA Lewis Research Center. Individuals at Lewis had already been working on these topics $[9,10,13-15]$ and Lewis had experience working with far-future ideas through their "Vision-21" exercises $[5,7,16]$. By applying the lessons learned from Vision-21 and by forging collaborations amongst the individuals across the country who were already working on these topics, Lewis established the "Breakthrough Propulsion Physics" program to advance science to address the goals of breakthrough space flight.

\section{PROGRAM FOUNDATIONS}

As the name implies, this program is specifically looking for propulsion breakthroughs from physics. It is not looking for further technological refinements of existing methods. Such refinements are being explored in other programs under the ASTP. Instead, this program looks beyond the known methods, searching for further advances in science from which genuinely new technology can emerge - technology to surpass the limits of existing methods.

There is a historical pattern to technological revolutions, where new methods surpass the fundamental limits of their predecessors [17]. Steam ships surpassed sailing ships, aircraft surpassed ground transportation, rockets surpassed aircraft, and now the

\footnotetext{
${ }^{1}$ Leader Breakthrough Propulsion Physics Program, marc.g.millis@lerc.nasa.gov, (216) 977-7535, Fax-7545
} 
search has begun for new methods to surpass rockets. This evolutionary pattern is summarized in Figure 1. To sustain technological preeminence, new methods must be sought when the existing method is reaching the limits of its underlying physical principles (the upper right asymptote of the S-curve in Figure 1), and when new clues are emerging for alternative methods that might surpass these limits [17].

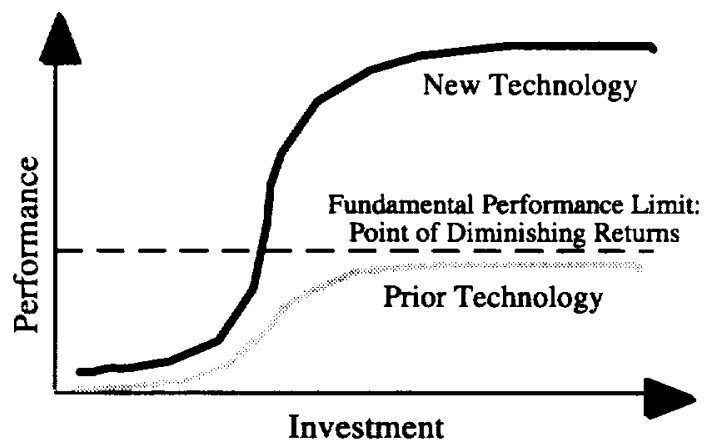

Fig. 1 S-Curve Pattern of Technology Revolution (Adapted from Foster, 1986)

In the case of spaceflight, rocket technology is reaching the performance limits of its underlying physical principles and new clues are emerging from science that might lead to new propulsion principles.

There have been several recent advances in science that have reawakened consideration that new propulsion mechanisms may lie in wait of discovery. Recent experiments and Quantum theory have revealed that space may contain enormous levels of vacuum electromagnetic energy $[18,19]$. This has led to questioning if this vacuum energy can be used as an energy source $[20,21,11]$ or a propulsive reaction mass for space travel [22]. Next, new theories suggest that gravity and inertia are electromagnetic effects related to this vacuum energy $[23,24]$. It is known from observed phenomena and from the established physics of General Relativity that gravity, electromagnetism, and spacetime are inter-related phenomena [25]. These ideas have led to questioning if gravitational or inertial forces can be created or modified using electromagnetism [22]. Also, theories have emerged from General Relativity about the nature of spacetime that suggest that the light-speed barrier, described by Special Relativity, might be circumvented by altering spacetime itself. These "wormhole" [26, 27] and "warp drive" theories [28, 29] have reawakened consideration that the light-speed limit of space travel may be circumvented. Today, it is still unknown whether these emerging theories are correct and, even if they are correct, if they can become viable candidates for creating propulsion breakthroughs.

Although these emerging possibilities are of keen interest to space technologists, the general scientific community is more concerned with answering questions of the origin of the universe, missing matter, black holes, and high-energy particle interactions. To advance physics to solve the challenges of space travel a focused effort is required. It should also be pointed out that such an application-oriented program also provides new opportunities for science itself. In the first step of the scientific method, where one clearly formulates the problem to guide the search for knowledge, this NASA program has a unique problem: space flight. This program is specifically interested in the physics of how to propel a space vehicle as far and as fast as possible with the least amount of effort. Such a focus will present different lines of inquiry than the more general physics inquiries. By asking different questions and looking along a different path, this program provides an opportunity for physicists to search for discoveries that may otherwise be overlooked or delayed.

Since such work is more visionary than usual aerospace endeavors, this program faces special programmatic challenges in addition to the technical challenges of discovering the desired breakthroughs. Fortunately, much has been written about the historical lessons from technological revolutions [17], scientific revolutions [30], and the human creative process [31]. Many of these lessons were incorporated into the NASA Lewis "Vision-21" activities [16], and have been incorporated into the Breakthrough Propulsion Physics program. In the descriptions of the program's goals, objective, methods, and research priorities that follow, these lessons are presented.

\section{3.l Program Goals}

The first step toward solving a problem is to define the problem. To determine the specific technical goals of the program, the "Horizon Mission Methodology" [32] was used. This method forces paradigm shifts beyond extrapolations of existing technologies by using impossible hypothetical mission goals to solicit new solutions. By setting impossible goals, the common practice of limiting visions to extrapolations of existing solutions is prevented. The "impossible" goal used in this exercise was practical interstellar travel. From conducting this exercise, the three major barriers to practical interstellar travel were identified and then set as the program's technical goals. These are the breakthroughs required to revolutionize space travel and enable interstellar voyages:

(1) MASS: Discover new propulsion methods that eliminate or dramatically reduce the need for propellant. This implies discovering fundamentally new ways to create motion, presumably by manipulating inertia, gravity, or by any other interactions between matter, fields, and spacetime.

(2) SPEED: Discover how to attain the ultimate achievable transit speeds to dramatically reduce travel times. This implies discovering a means to move a vehicle at or near the actual maximum speed limit for motion through space or through the 
motion of spacetime itself (if possible, this means circumventing the light speed limit).

(3) ENERGY: Discover fundamentally new modes of onboard energy generation to power these propulsion devices. This third goal is included since the first two breakthroughs could require breakthroughs in energy generation, and since the physics underlying the propulsion goals is closely linked to energy physics.

\subsection{Program Objective}

The objective of the NASA Breakthrough Propulsion Physics Program is to produce near-term, credible, and measurable progress toward conquering these three goals. The underlined terms are some of the programmatic features needed to conduct such visionary work in formal institutions such as NASA.

The emphasis on "near-term progress" is because the program's goals are presumably far from fruition while the support for the program is sought in the near-term. It is therefore essential that the long-range goals be broken down into smaller, near-term steps. This is reflected in the Research Priorities discussed later.

Closely related to the need for near-term progress, is the need to measure this progress. The program's sponsors want to see progress within the funding cycles. The Research Priority criteria, discussed later, include means to quantify progress.

The emphasis on "credible" is because such long range ambitions are often tainted by non-credible work, or even "pathological science" [33, 34], and since genuine progress can only be made with credible work. The challenge to balance credibility (necessary to make genuine progress) with vision (necessary to search beyond known methods) is also addressed in the Research Priorities discussed later. Another aspect of credibility is that this program does not promise to deliver the breakthroughs, but does promise to deliver progress toward achieving the breakthroughs. This position is because it is too soon to know if the desired breakthroughs are indeed achievable.

\subsection{Collaborative Networking}

Historically, pioneering new ideas has often been the jurisdiction of exceptional individuals who not only possessed the vision to realize their creations, but also the determination to weather the setbacks, the skills to translate their ideas into credible proofs-of-concepts, and the ability to make others comprehend their creations. Individuals who posses all these skills at once are rare, but this skill mix often exists in a group of individuals. By providing a means for these individuals to collaborate and share their skill mix to achieve a common goal, pioneering work can proceed without having to wait for the next Goddard or Einstein.
This program was born out of the collaborative networking of individual researchers who explored such topics out of their own interests. This program will continue such collaborative networking. This networking is open to all the NASA centers, government labs, universities, and industries, and credible individuals. Also, this program has recently opened up this collaboration to the international community. Collaborative networking has the following advantages:

- A diverse, multidisciplinary team provides a well rounded and more objective program.

- Expertise and talent are scattered across the world, and are not centralized at a single lab.

- Collaboration boosts credibility.

- Collaboration opens the way for collateral support (where researchers seek support from their host organizations while retaining open information exchange).

- Collaboration allows phased peer reviews, first with the constructive team, then with external reviewers.

The internet is envisioned as the primary mechanism to enable this degree of collaboration and to pool the collective intellect across the world. Two internet sites have already been set up, and a third is envisioned. One site, the "Warp Drive, When?" site (http://www.lerc.nasa.gov/WWW/PAO/warp.htm), is for public education. It describes the difficulties and emerging possibilities of interstellar travel. The second site, the Breakthrough Propulsion Physics Program site (http://www.lerc.nasa.gov/WWW/bpp/), lists the details of this program and its status. The third site is envisioned to be a limited access site. It will contain works in progress, more in-depth annotated bibliographies, and allow on-line discussions. Access will be limited to a "Contributor Network" of researchers selected by the program's government member steering group. This limited access site has not yet been completed, nor has the process for nominating and selecting Contributor Network members been specified.

Another means to allow collaborative networking is through conferences and workshops. The following is a list of the sessions and workshops held and planned that are related to this topic:

- Feb. 97, Brainstorming Meeting, Austin TX.

- Aug. 97, Breakthrough Propulsion Physics Workshop, Cleveland OH [35].

- Jan. 98, STAIF, 2 sessions, Albuquerque, NM.

- Jun. 98, IAA Symposium, Aosta ITALY.

- Jul. 11,98, AIAA Joint Propulsion Conference, 1 session, Cleveland, $\mathrm{OH}$.

- Jan. 99, STAIF, 3 sessions, Albuquerque, NM.

- Spring 99, Breakthrough Propulsion Physics Workshop \# 2 (in planning).

- Jul. 99, AIAA Joint Propulsion Conference, 1 session, Los Angeles, CA. 


\subsection{Supporting Research}

Presently, this program has only received enough funds to conduct the kick-off workshop and establish the web sites. Efforts are underway to secure funding to formally solicit and support research tasks. In the interim, and for international researchers that are not eligible for US funding, researchers are encouraged to seek funding through their own host organizations. With the precedent of this NASA program, and by using this program's Research Priorities as a guide, it may now be easier for other researchers to secure funding for such visionary work.

Recently the NASA "Small Business Innovative Research (SBIR)" and "Space Technology Transfer Research (STTR)" funding mechanisms have had breakthrough propulsion added to their solicitation topics (http://sbir.gsfc.nasa.gov/). Researchers are encouraged to investigate these alternative funding mechanisms.

Once funded, this program plans to use an annual "NASA Research Announcement" (NRA) to solicit and support research tasks. This solicitation will be open to academia, industry, government labs, and NASA centers. Selection will be via a peer review process using the Research Prioritization Criteria to provide an initial ranking. Because it is too early to focus on a given approach, it is anticipated that multiple, different approaches will be supported from the top ranking candidates. Proposed tasks should be of relatively short duration (1-3yrs), modest cost $(\$ 50$ to $\$ 150 \mathrm{~K})$, and traceable to at least one of the three program goals.

\section{RESEARCH PRIORITIES}

To simultaneously focus emerging sciences toward answering the needs of space travel and to provide a programmatic tool for measuring the relative value and progress of research, this program has established the prioritization criteria listed below. This evaluation system has already gone through three iterations including two trial runs. A derivative of this system is planned as the scoring system for the program's NRA solicitation. The features of the system that are discussed in this report include: (1) near-term focus on long range goals, (2) metrics of progress, and (3) credibility criteria with vision.

\subsection{Research Prioritization Criteria List:}

This list shows those factors that would be scored to measure the relative value and progress of research. Each of the lettered criteria below would receive a numeric score which would then be combined to arrive at a total score for a given research approach.

- Relevance To Program:

A. Directness (must seek advances in physics that are relevant to propulsion or power).

B. Magnitude of potential gains for goal \#1 (mass)

+ goal $\# 2$ (speed) + goal \#3 (energy).
- Readiness:

C. Level of progress achieved to date (measured using the scientific method levels).

D. Testability (ease of empirical testing).

[Note: experiments are considered closer than theory to becoming technology].

- Credibility: [Note: these are designed to insure credibility while still being open to visionary ideas]

E. Fits credible data (references must be cited).

F. More advantageous to program goals than current approaches (references of competing approaches must be cited).

G. Discriminating test suggested.

- Research Task Factors:

H. Level of progress to be achieved upon completion of task (measured using the scientific method levels).

I. Breadth of work (experiment, theory, and/or comparative study).

J. Triage (will it be done anyway or must this program support it?).

K. Lineage (will it lead to further relevant advancements?).

L. Time required to complete task (reciprocal scoring factor).

M. Funding required (reciprocal scoring factor).

N. Probability of successful task completion (based on credentials and realism of proposal).

\subsection{Near-Term Focus to Long-Range Goals}

The program's goals are presumably far from fruition while the support for the program is sought in the nearterm. To address this paradox it is essential that the long-range goals be broken down into smaller, affordable, near-term steps. Proposals are therefore required to suggest only an incremental task related to the ultimate goals, and are graded inversely to their duration and cost (criteria $\mathrm{L}$ and $\mathrm{M}$ ). Also, from this point of view, "success" is defined as learning more about reaching the breakthrough, rather than actually achieving the breakthrough. Negative test results are still results, indicating progress.

\subsection{Metrics of Progress}

Closely related to the focus on near-term steps, is the need to measure progress. To demonstrate to the program sponsors that progress is being made in the short time-frame of funding cycles, these Prioritization Criteria can be used to quantify progress. By simply taking the difference in score before and after a task is completed, a numerical value of "progress" can be calculated. Since there is no precedent for such a system, these values will only have meaning when comparing the progress of different tasks over different years.

One crucial feature inherent in this system is to have a scale to gauge the status of an approach. Patterned after the "Technology Readiness Scale" used to 
compare engineering status, the Scientific Method has been adapted to address the science that precedes technology. This scale, listed below in order of increasing maturity, are used in criteria $\mathrm{C}$ and $\mathrm{H}$. For scoring, a numeric value would be assigned to each level based roughly on an estimate of the relative quantity of work to achieve that level.

- Sci. Method Step $\varnothing$ : Pre Science - recognizing an opportunity.

- Sci. Method Step 1: Problem Formulated.

- Sci. Method Step 2: Data Collected.

- Sci. Method Step 3: Hypothesis Proposed.

- Sci. Method Step 4: Hypothesis Tested \& Results Reported.

- Tech Readiness Level 1: Basic Principles Observed \& Reported, same as Sci. Step 4.

- Tech Readiness Level 2: Applications Conceptual Design Formulated.

\subsection{Balancing Credibility With Vision}

Another challenge of seeking breakthroughs is ensuring credibility without sacrificing openness to new perspectives. This is particularly challenging since genuinely new ideas often extend beyond the established knowledge base, or worse, can appear to contradict this base. In other words, a genuinely new, credible idea is very likely to appear non-credible. Also, it is common when soliciting new ideas to receive a large number of "fringe" submissions that are certainly non-credible. To address this challenge, it is recommended to: (1) concentrate on credible empirical data (how nature is observed to work) rather than depending on current theories or paradigms (how nature is interpreted to work), (2) compare the new idea's value to existing approaches, (3) ensure that the new idea can be put to a test, and (4) look for the characteristic signs of non-credible science [34]. It should be noted that these credibility criteria do not check if an idea is correct, but rather check to see if the idea is credibly constructed and is leading to a correctness test.

Some of the characteristics of non-credible work is that references are not explicitly cited, and that conclusions are made without substantiating the work with supporting evidence. This can be easily checked by requiring that submissions cite credible, peer reviewed, references. References are required for supporting evidence (criteria E), and for comparisons to existing theories (criteria F). Fringe or pathological researchers often do not do this homework. These credibility checks still leave plenty of room for unconventional, visionary ideas.

Empiricism is emphasized over theory as a credibility check since theory is an interpretation to explain observations of nature - our current best perspective. Theories evolve over time as we gain more understanding about nature, but the empirical observations, the raw data, do not change. For example, the data of the motions of the planets are the same, regardless if one uses the Copernicus theory or the Earth-centered theory to describe the data. When seeking new ideas, it is crucial that they are consistent with credible data, but they may entertain new interpretations of that data. This emphasis of empiricism over theory is the primary technique to allow credible vision.

To ensure that the idea is oriented toward the goals of the program, and to ensure that the author has done their homework, it is required that the proposal articulate how the new idea compares to existing approaches (criteria F). This not only checks for relevance and to insure reference citations, as mentioned before, but positions the idea to address the next critical criteria; a discriminating test.

A discriminating test (criteria $G$ ) is required to focus the work toward the make-or-break issues, and to provide the basis for a credible "correctness" test.

\section{AUGUST 1997 WORKSHOP}

One of the first major milestones of the program was to convene a workshop with established physicists, government researchers and select innovators to jointly examine the new theories and phenomena in the context of seeking propulsion breakthroughs. This workshop was held on August 12-14, 1997, in Cleveland Ohio [35].

The purpose of the workshop was to understand the fundamental issues and opportunities for new propulsion physics and to foster collaborations amongst researchers. A key deliverable was to assemble a list of candidate research tasks. To achieve this purpose, this workshop featured a plenary sequence of 14 invited presentations about emerging physics with both optimistic and pessimistic viewpoints, 30 poster papers for provoking thought, and 6 parallel breakout sessions for the participants to generate a list of next-step research tasks.

Since this workshop dealt with seeking breakthroughs in science, it asked participants to be visionary. Admittedly, these breakthroughs may turn out to be impossible, but progress is not made by conceding defeat. For the sake of promoting progress, participants were asked to entertain, for the duration of the workshop, the notion that these breakthroughs are indeed achievable. Simultaneously, however, this workshop looked for sound and tangible research approaches. Therefore, participants were also asked to be credible -- credible progress toward incredible possibilities.

In total, 84 participants attended the workshop, including 26 from industry, 18 from universities, 12 from six government labs, 16 from five NASA centers, and 12 students.

\subsection{Invited Presentations}

The invited presentations, from established physicists, covered many of the relevant areas of emerging 
physics. The intent of these presentations was to provide credible overviews of where we stand today in physics and introduce the unknowns and unresolved issues. Below is a list of these presentations in the order that they were presented. Where a related or equivalent work is available, a reference is cited.

(1) L. Krauss (Case Western Reserve Univ.), "Propellantless Propulsion: The Most Inefficient Way to Fly?" [36]

(2) H. Puthoff (Inst. for Advanced Studies at Austin), "Can the Vacuum be Engineered for Spaceflight Applications?: Overview of Theory and Experiments" $[11,21,23,24]$

(3) R. Chiao (Univ. of California at Berkeley) \& A. Steinberg, "Quantum Optical Studies of Tunneling Times and Superluminality" [37]

(4) J. Cramer (Univ. Washington), "Quantum Nonlocality and Possible Superluminal Effects" [38]

(5) R. Koczor \& D. Noever (MSFC), "Experiments on the Possible Interaction of Rotating Type II $Y B C O$ Ceramic Superconductors and the Local Gravity Field" $[39,40]$

(6) R. Forward (Forward Unlimited), "Apparent Endless Extraction of Energy from the Vacuum by Cyclic Manipulation of Casimir Cavity Dimensions" $[41,20]$

(7) B. Haisch (Lockheed) \& A. Rueda, "The ZeroPoint Field and the NASA Challenge to Create the Space Drive" [24]

(8) A. Rueda (California State Univ.) \& B. Haisch, "Inertial Mass as Reaction of the Vacuum to Accelerated Motion" [24]

(9) D. Cole (IBM Microelectronics), "Calculations on Electromagnetic Zero-Point Contributions to Mass and Perspectives" [21].

(10) P. Milonni (Los Alamos), "Casimir Effect: Evidence and Implications" [18]

(11) H. Yilmaz (Electro-Optics Tech. Ctr.), "The New Theory of Gravitation and the Fifth Test" [42]

(12) A. Kheyfets (N. Carolina St. U.) \& W. Miller, "Hyper-Fast Interstellar Travel via Modification of Spacetime Geometry" [26-29, 43].

(13) F. Tipler, III (Tulane U.), "Ultrarelativistic Rockets and the Ultimate Future of the Universe"

(14) G. Miley (U. of Illinois), "Possible Evidence of Anomalous Energy Effects in H/D-Loaded Solids- Low Energy Nuclear Reactions"

\subsection{Identifying Next-Step Research Tasks}

To generate the list of next-step research tasks, the participants were divided into six breakout groups. Each of the three program goals were addressed by two of the six groups. A facilitator led the group through a process designed to elicit a large number of ideas and then to evolve these ideas into candidate next-step research tasks - tasks that address the immediate questions raised by the emerging physics and the program goals. To be programmatically acceptable, it was desired that these research tasks be short-duration, low-cost, and incremental steps toward the grand goals. Based on the invited presentations, poster papers, and the ideas generated during the breakout sessions, about 80 task ideas were collected.

\section{CANDIDATE NEXT-STEP RESEARCH}

The following section highlights just some of the approaches that have been suggested to begin the search for propulsion breakthroughs. These are arranged according to the three program goals and highlight the intriguing phenomena and theories, critical issues, and candidate next-step approaches for each program goal. Some of the 48 ideas that were generated during the Austin Texas brainstorming session, and some of the 80 ideas from the August workshop hare covered here. Note that there are many redundancies amongst these 128 ideas, and that most of these have not yet been fully reviewed.

\subsection{Toward Eliminating Propellant Mass}

It is known that gravity, electromagnetism and spacetime are coupled phenomena. Evidence includes the bending of light, the red-shifting of light, and the slowing of time in a gravitational field. This coupling is most prominently described by General Relativity [25]. Given this coupling and our technological proficiency for electromagnetics, it has been speculated that it may become possible to use electromagnetic technology to manipulate inertia, gravity, or spacetime to induce propulsive forces [22]. Another phenomena of interest is the Casimir Effect, where closely spaced plates are forced together, presumably by vacuum fluctuations [19]. One explanation is that this force is the net radiation pressure of the virtual vacuum fluctuation photons, where the pressure is greater outside the plates than within, since wavelengths larger than the plate separation are excluded. The force is inversely proportional to the 4th power of the distance. Even though this effect can be explained by various theories [18], the idea that the vacuum might create these forces leads to speculations that an asymmetric vacuum effect, if possible, could lead to a propulsive effect [22]. There are many unsolved issues regarding these speculations, including whether these phenomena can lead to controllable net-force effects and whether such effects can be created, even in principle, without violating conservation of momentum and energy [22].

Although it is presently unknown if such propellantless propulsion can be achieved, several theories have emerged that provide additional research paths. It should be noted that all of these theories are too new to have either been confirmed or discounted, but their potential utility warrants consideration. This includes negative mass propulsion [44], theories that suggest that inertia and gravity are affected by vacuum fluctuations $[23,24]$ and numerous other theories about 
the coupling between matter, electromagnetism, and spacetime $[4,42,45-50]$. Another recent development, which has yet to be credibly confirmed or discounted, is where anomalous weight changes are observed over spinning superconductors [39].

Regarding candidate next steps, experiments have been suggested to test most of the theories cited above, including the theories linking inertia to vacuum fluctuations [11]. Furthermore, Robert Forward suggested a search for negative mass based on recent astronomical data [51]. Also, experiments at MSFC are continuing to test the claims of weight changes over spinning superconductors [40].

\subsection{Toward Achieving the Ultimate Transit Speed}

Special Relativity states that the speed of light is an upper limit for the motion of matter through spacetime. Recently, however, theories using the formalism of General Relativity have suggested that this limit can be circumvented by altering spacetime itself. This includes "wormhole" and "warp drive" theories. A wormhole is a shortcut created through spacetime [26, 27] where a region of spacetime is warped to create a shorter path between two points. A warp drive involves the expansion and contraction of spacetime to propel a region of spacetime faster than light [28]. Figure 2 illustrates the Alcubierre warp drive, showing the opposing regions of expanding and contracting spacetime that propel the center region.

It has also been suggested that the light speed limit may be exceeded if velocities could take on imaginary values [52]. In addition, there are theories for "nonlocality" from Quantum Physics that suggest potentially superluminal effects [38]. These theories not only present challenging physics problems, but are intriguing from the point of view of future space travel. Do these theories represent genuinely possible physical effects, or are they merely mathematical curiosities?

Wormholes, if they exist, may be observable through astronomical searches. The characteristic signature of a negative mass wormhole (possibly a traverseable type) has been specified to aid this search [53]. Regarding possible experiments, it has been suggested to use the strong magnetic fields that are momentarily generated by chemical and nuclear explosions and lasers to test the space-warping effect of magnetic fields [54].

Regarding other faster-than-light possibilities, there have also been some intriguing experimental effects. Photons have been measured to tunnel across a photonic band-gap barrier at 1.7 times the speed of light [37]. Even though the author concludes that information did not travel faster than light, the results are intriguing. It has been suggested to conduct similar experiments using matter rather than photons to unambiguously test the information transfer rate. In addition, recent experiments of the rest mass of the electron antineutrino have measured an imaginary value [55]. Even though this result is attributed to possible errors, an imaginary mass value could be a signature characteristic of a tachyon (hypothetical faster-thanlight particles). It has been suggested to revisit this and other similar data to determine if this can be credibly interpreted as evidence of tachyons. It was also pointed out that other experiments have been suggested to search for evidence of tachyons [56].

The notion of faster-than-light travel evokes many critical issues. Issues include causality violations, the requirement for negative energy, and the requirement for enormous energy densities to create the superluminal effects. Theoretical approaches have been suggested to address these issues, including the use of quantum gravity.

\subsection{Toward New Modes of Energy Production}

Since the first two breakthroughs could require breakthroughs in energy generation, and since the physics underlying the propulsion goals is closely linked to energy physics, it is also of interest to discover fundamentally new modes of energy generation. The principle phenomena of interest for this category is, again, the vacuum fluctuations. It has been theorized that this energy can be extracted without violating conservation of energy or any thermodynamic laws $[20,21]$. It is still unknown if this vacuum energy exists as predicted, how much energy might be available to extract, and what the secondary consequences would be of extracting vacuum energy.

It has been suggested to continue experimental work

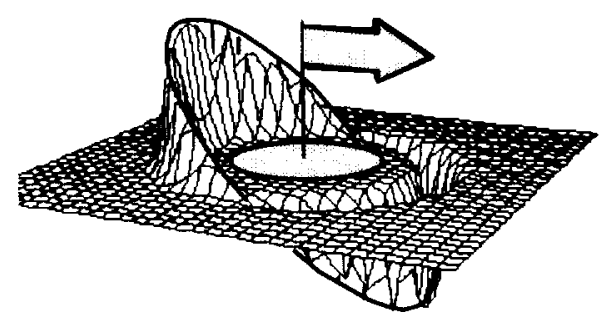

Figure 2 The Alcubierre Warp Drive

to study the Casimir effect, not only to address these energy questions, but to explore the more general physics of geometry and temperature effects on the Casimir effect. Techniques have been suggested for using micromechanical technology to study Casimir effects [57]. Not only are micromechanical structures an emerging technology, but the dimensions of such structures are similar to the dimensions required for Casimir effects. Also, should any viable device be engineered, these methods might be adaptable for highvolume manufacturing. On another vein, it has been suggested to continue the study of the sonoluminescence effect and its relation to vacuum fluctuation energy [58].

On a more conventional vein, ideas were raised at the workshop by Tipler and LaPointe for seeking alternative methods of antimatter production. 


\section{CONCLUSIONS}

New theories and laboratory-scale effects have emerged in the scientific literature which provide new approaches to seeking major propulsion breakthroughs. NASA has established a program to begin exploring these possibilities. Since the propulsion goals are presumably far from fruition, a special emphasis of the program is to identify affordable, near-term, and credible research that could make measurable progress toward these propulsion goals. To kick-off the program, collaborative networking, internet communication, and workshops are being used. During a recent workshop, many of these new approaches were reviewed, and several research task ideas were generated for taking the next steps toward propulsion breakthroughs. A NASA Research Announcement has been chosen as the mechanism to solicit and support research, once sufficient funds become available. A peer review system has been drafted to rank these and other future proposals. In the interim, other funding opportunities such as the SBIR and STTR are available.

\section{REFERENCES}

[1] Mead, F. Jr. (1989) "Exotic Concepts for Future Propulsion and Space Travel", Advanced Propulsion Concepts, 1989 JPM Specialist Session, (JANNAF), CPIA Publication 528:93-99.

[2] Evans, R. A., ed. (1990) BAe University Round Table on Gravitational Research, Report on Meeting held in Preston UK, March 26-27, 1990, Report \# FBS 007, British Aerospace Limited, Preston, UK.

[3] Forward, R. L. (1990) "21st Century Space Propulsion Study," Report \# AL-TR-90-030, Air Force Astronautics Lab (AFSC), Edwards AFB, CA.

[4] Cravens D. L. (1990) "Electric Propulsion Study," Report \# AL-TR-89-040, Air Force Astronautics Lab (AFSC), Edwards AFB, CA

[5] Landis, G. L., ed. (1990) "Vision-21: Space Travel for the Next Millennium" Proceedings, NASA Lewis Research Center, April 3-4 1990, NASA CP 10059 , NASA Lewis Research Center.

[6] Talley, R. L. (1991) "Twenty First Century Propulsion Concept," Report \# PL-TR-91-3009, Phillips Laboratory, Air Force Systems Command, Edwards AFB, CA.

[7] Landis, G., and Bailey, S. ed. (1993) "Vision-21: Interdisciplinary Science and Engineering in the Era of Cyberspace" Proceedings, NASA Lewis Research Center, March 30-31 1993, NASA CP 10129, NASA Lewis Research Center.

[8] Bennett, G., Forward, R. L. and Frisbee, R. (1995) "Report on the NASA/JPL Workshop on Advanced Quantum/Relativity Theory Propulsion" AIAA 95-2599, 31 st AIAA/ASME/SAE/ASEE Joint Propulsion Conference.

[9] Millis, M. and Williamson, G. S. (1995) "Experimental Results of Hooper's Gravity-Electromagnetic Coupling Concept," NASA TM 106963, Lewis Research Center.

[10] Niedra, Myers, Fralick, \& Baldwin (1996) "Replication of the Apparent Excess Heat Effect in a Light WaterPotassium Carbonate-Nickel Electrolytic Cell" NASA TM 107167, NASA LeRC.
[11] Forward, R. L. (1996) "Mass Modification Experiment Definition Study," Report \# PL-TR-96-3004, Phillips Lab, Edwards AFB, CA.

[12] Bachtel, F. D. and Lyles, G. M. (1997) "A Technology Plan for Enabling Commercial Space Business," AIF97-V.4.02, 48th International Astronautical Congress, Oct. 6-10, 1997, Turin Italy.

[13] Zampino, E. (1986) "A Brief Study on the Transformation of Maxwell Equations in Euclidian Four-Space", In J. Math. Physics, 27:1315-1318.

[14] Millis, M. (1990) "Exploring the Notion of Space Coupling Propulsion", In NASA Lewis Research Center CP 10059, NASA. pp. 307-316.

[15] Landis, G. (1991) "Comments on Negative Mass Propulsion," In J of Propulsion and Power, 7:304.

[16] Millis, M. (1990) "Speculating on Space Futures," Space Policy, 6:353-356.

[17] Foster, R. N. (1986) Innovation; The Attacker's Advantage, Summit Books.

[18] Milonni, P. W. (1994) The Quantum Vacuum, Academic Press, San Diego, CA.

[19] Lamoreaux, S. K. (1997) "Demonstration of the Casimir Force in the 0.6 to $6 \mu \mathrm{m}$ Range," Phys. Rev. Letters, 78:5-8.

[20] Forward, R. L. (1984) "Extracting Electrical Energy from the Vacuum by Cohesion of Charged Foliated Conductors," Physical Review B, 15 AUG. 1984 B30:1700-1702.

[21] Cole, D. and Puthoff. (1993) "Extracting Energy and Heat from the Vacuum," Phys Rev E, 48:1562-1565.

[22] Millis, M. (1997b) "Challenge to Create the Space Drive," $J$ of Propulsion and Power, 13:577-582.

[23] Puthoff, H. E. (1989) "Gravity as a zero-pointfluctuation force," Phys Rev A, 39:2333-2342.

[24] Haisch, B., Rueda, A., and Puthoff, H. E. (1994) "Inertia as a Zero-Point Field Lorentz Force," Physical Review A, 49:678-694.

[25] Misner C. W., Thorne, K. W., and Wheeler, J. A. (1973) Gravitation, W. H. Freeman \& Company, NY.

[26] Morris, M. and Thorne, K. (1988) "Wormholes in Spacetime and Their Use for Interstellar Travel: A Tool for Teaching General Relativity," American Journal of Physics, 56:395-412.

[27] Visser, M. (1995) Lorentzian Wormholes - From Einstein to Hawking, AIP Press, Woodbury, NY.

[28] Alcubierre, M. (1994) "The Warp Drive: Hyper-fast Travel Within General Relativity," Classical and Quantum Gravity, 11:L73-L77.

[29] Krasnikov, S. V. (1995) "Hyper-Fast Interstellar Travel in General Relativity," gr-qc, 9511068.

[30] Kuhn, T. S. (1970) The Structure of Scientific Revolutions, 2nd Ed. Univ. of Chicago Press, Chicago.

[31] Miller, W. C. (1987) The Creative Edge: Fostering Innovation Where You Work, 2nd printing. AddisonWesley Publishing Co. Inc., Reading MA.

[32] Anderson, J. L. (1996) "Leaps of the Imagination: Interstellar Flight and the Horizon Mission Methodology," JBIS, 49:15-20.

[33] Taubes, G. (1993) Bad Science - the Short Life and Weird Times of Cold Fusion, Random House, NY.

[34] Bennett, G. L. (1997) "Some Observations on Avoiding Pitfalls in Developing Future Flight Systems" AIAA 97. 3209, 33rd AIAA/ASME/SAE/ ASEE Joint Propulsion Conference. 
[35] Millis, M. (1998) "Breakthrough Propulsion Physics Workshop Preliminary Results," Space Technology and Applications International Forum, AIP Conference Proceedings 420:3-12, Albuquerque NM (Jan. 98), and NASA TM-97-206241 (Nov. 97).

[36] Krauss, L. M. (1995) The Physics of Star Trek, Basic Books, NY.

[37] Chiao, R. Y., Steinberg, A. M., and Kwiat, P. G. (1994) "The Photonic Tunneling Time and the Superluminal Propagation of Wave Packets," Proc. of the Adriatico Workshop on Quantum Interferometry, DeMartini, Denardo, and Zeilinger, eds., World Scientific, Singapore, p. 258.

[38] Cramer, J. G. (1986) "The Transactional Interpretation of Quantum Mechanics," Reviews of Modern Physics, A. Phys. Soc., 58:647-688.

[39] Podkletnov, E. and Nieminen, R. (1992) "A Possibility of Gravitational Force Shielding by Bulk $\mathrm{YBa}_{2} \mathrm{Cu}_{3} \mathrm{O}_{7}$. x Superconductor," Physica C, C203:441-444.

[40] Li, N., Noever, D., Robertson, T., Koczor, R., and Brantley, W. (1997) "Static Test for a Gravitational Force Coupled to Type II YBCO Superconductors," Physica C, 281:260-267.

[41] Ambjørn, J. and Wolfram, S. (1983) "Properties of the Vacuum, 1. Mechanical and Thermodynamic, and Properties of the Vacuum, 2. Electrodynamic," Annals of Physics, 147:1-56.

[42] Yilmaz, H. (1992), "Toward a Field Theory of Gravitation," Il Nuovo Cimento, 107B:941-960.

[43] Pfenning, M., Ford, L. (1997) "The Unphysical Nature of Warp Drive," $g r-q c, 9702026$.

[44] Bondi, H. (1957) "Negative Mass in General Relativity," Reviews of Modern Physics, 29:423-428.

[45] Vargas, J. (1991) "On the Geometrization of Electrodynamics," Found. of Phy.s, 21:379-401.

[46] Ringermacher, H. (1994) "An Electrodynamic Connection," Classical and Quantum Gravity, 11:2383. 2394.

[47] Woodward, J. F. (1994) "Method for Transiently Altering the Mass of an Object to Facilitate Their Transport or Change their Stationary Apparent Weights," US Patent 5,280,864.
[48] Brandenburg, J. E. (1995) “A Model Cosmology Based on Gravity-Electromagnetism Unification," Astophysics and Space Science, 227:133-144.

[49] Schlicher R. L., Biggs, A. W., and Tedeschi, W. J. (1995) "Mechanical Propulsion From Unsymmetrical Magnetic Induction Fields", AIAA 95-2643, 31 st AIAA/ASME/SAE/ASEE Joint Propulsion Conf.

[50] Froning, H. D. and Barrett, T. W. (1997) "Inertial Reduction and Possible Impulsion by Conditioning Electromagnetic Fields," AIAA 97-3170, 33rd AIAA/ASME/SAE/ASEE Joint Prop. Conference.

[51] da Costa, L. N., Freudling, W., Wegner, G., Giovanelli, R., Haynes, M. P., and Salzer, J. J. (1996) "The Mass Distribution in the Nearby Universe," Astrophysical Journal Letters, 468:L5-L8 and L1.

[52] Asaro, C. (1996) "Complex Speeds and Special Relativity," Am. J. Phys., 64(4):412-429.

[53] Cramer J., Forward, R. L., Morris, M., Visser, M., Benford, G. and Landis, G. (1994) "Natural Wormholes as Gravitational Lenses," Physical Review D, 15 March 1995:3124-3127.

[54] Davis, E. W. (1998) "Interstellar Travel by Means of Wormhole Induction Propulsion (WHIP)," Space Technology and Applications International Forum, AIP Conference Proceedings 420:1502-1508, Albuquerque NM (Jan. 1998).

[55] Stoeffl, W. and Decman D. J. (1995) "Anomalous Structure in the Beta Decay of Gaseous Molecular Tritium," Physical Review Letters, 75:3237-3240.

[56] Chiao, R. Y., Kozhekin, A. E., and Kurizki G. (1996) "Tachyonlike Excitations in Inverted Two-Level Media," Phys. Rev. Lett. 77:1254

[57] Serry, F. M., Walliser, D., Maclay, G. J. (1995) "The Anharmonic Casimir Oscillator," $J$. Microelectromechanical Systems, 4:193.

[58] Eberlein, C. (1996) "Theory of quantum radiation observed as sonoluminescence," Phys Rev A, 53:27722787. 


\begin{tabular}{|c|c|c|c|c|}
\hline \multicolumn{3}{|c|}{ REPORT DOCUMENTATION PAGE } & \multicolumn{2}{|r|}{$\begin{array}{l}\text { Form Approved } \\
\text { OMB No. 0704-0188 }\end{array}$} \\
\hline \multicolumn{5}{|c|}{ 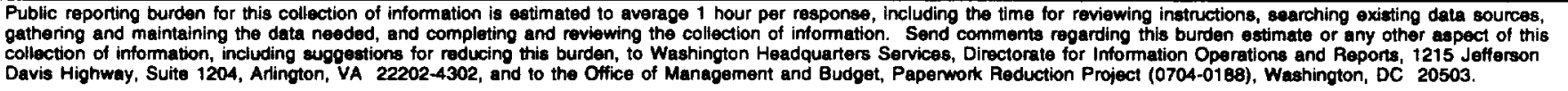 } \\
\hline 1. AGENCY USE ONLY (Leave blank) & $\begin{array}{r}\text { 2. REPOFT DATE } \\
\text { June } 1998\end{array}$ & \multicolumn{3}{|c|}{$\begin{array}{l}\text { 3. REPORT TYPE AND DATES COVERED } \\
\text { Technical Memorandum }\end{array}$} \\
\hline \multicolumn{3}{|c|}{$\begin{array}{l}\text { 4. TITLE AND SUBTTILE } \\
\text { NASA Breakthrough Propulsion Physics Program }\end{array}$} & \multicolumn{2}{|c|}{ 5. FUNDING NUMBERS } \\
\hline \multicolumn{3}{|l|}{$\begin{array}{l}\text { 6. AUTHOA(S) } \\
\text { Marc G. Millis }\end{array}$} & \multicolumn{2}{|c|}{ WU-953-74-40-00 } \\
\hline \multicolumn{3}{|c|}{$\begin{array}{l}\text { 7. PERFORMING ORGAMIZATION NAME(S) AND ADDRESS(ES) } \\
\text { National Aeronautics and Space Administration } \\
\text { Lewis Research Center } \\
\text { Cleveland, Ohio } 44135-3191\end{array}$} & \multicolumn{2}{|c|}{$\begin{array}{l}\text { B. PERFORMING ORGANIZATION } \\
\text { REPORT NUMBER } \\
\text { E-11231 }\end{array}$} \\
\hline \multicolumn{3}{|c|}{$\begin{array}{l}\text { 9. SPONSORINGMONITORING AGENCY NAME(S) AND ADDRESS(ES) } \\
\text { National Aeronautics and Space Administration } \\
\text { Washington, DC 20546-0001 }\end{array}$} & \multicolumn{2}{|c|}{$\begin{array}{l}\text { 10. SPONSORINGMONITORING } \\
\text { AGENCY REPORT NUMBER } \\
\text { NASA TM-1998-208400 }\end{array}$} \\
\hline \multicolumn{5}{|c|}{$\begin{array}{l}\text { 11. SUPPLEMENTAAY NOTES } \\
\text { Prepared for the Second Symposium on Realistic Near-Term Advanced Scientific Space Missions cosponsored by the } \\
\text { International Academy of Astronautics and Politecnico di Torino, Aosta, Italy, June 29-July 1, 1998. Responsible } \\
\text { person, Marc G. Millis, organization code } 5870,(216) 977-7535 .\end{array}$} \\
\hline \multicolumn{3}{|c|}{$\begin{array}{l}\text { 12a. DISTRIBUTIONAVAILABILTYY STATEMENT } \\
\text { Unclassified - Unlimited } \\
\text { Subject Category: } 70 \quad \text { Distribution: Nonstandard } \\
\text { This publication is available from the NASA Center for AeroSpace Information, (301) 621-0390 }\end{array}$} & \multicolumn{2}{|c|}{ 12b. DISTRIBUTION CODE } \\
\hline \multicolumn{5}{|c|}{$\begin{array}{l}\text { In 1996, NASA established the Breakthrough Propulsion Physics program to seek the ultimate breakthroughs in space } \\
\text { transportation: propulsion that requires no propellant mass, propulsion that attains the maximum transit speeds physically } \\
\text { possible, and breakthrough methods of energy production to power such devices. Topics of interest include experiments } \\
\text { and theories regarding the coupling of gravity and electromagnetism, vacuum fluctuation energy, warp drives and worm- } \\
\text { holes, and superluminal quantum effects. Because these propulsion goals are presumably far from fruition, a special } \\
\text { emphasis is to identify affordable, near-term, and credible research that could make measurable progress toward these } \\
\text { propulsion goals. The methods of the program and the results of the } 1997 \text { workshop are presented. This Breakthrough } \\
\text { Propulsion Physics program, managed by Lewis Research Center, is one part of a comprehensive, long range Advanced } \\
\text { Space Transportation Plan managed by Marshall Space Flight Center. }\end{array}$} \\
\hline \multirow{2}{*}{\multicolumn{4}{|c|}{ Space propulsion; Physics; General relativity; Gravity; Special relativity; Quantum physics }} & $\begin{array}{l}\text { 15. NUMBER OF PAGES } \\
15 \\
\end{array}$ \\
\hline & & & & $\begin{array}{l}\text { 16. PRICE CODE } \\
\text { A03 }\end{array}$ \\
\hline $\begin{array}{l}\text { 17. SECURTYY CLASSIFICATION } \\
\text { OF REPORT } \\
\text { Unclassified }\end{array}$ & $\begin{array}{l}\text { 18. SECUAITY CLASSIFICATION } \\
\text { OF THIS PAGE } \\
\text { Unclassified }\end{array}$ & $\begin{array}{l}\text { 19. SECURTYY CLASSIFIC } \\
\text { OF ABSTRACT } \\
\text { Unclassified }\end{array}$ & TION & 20. LIMTTATION OF ABSTRACT \\
\hline NSN 7540-01-280-5500 & & & & $\begin{array}{l}\text { andard Form } 298 \text { (Rev. 2-89) } \\
\text { ascribed by ANSI Sid. Z39-18 }\end{array}$ \\
\hline
\end{tabular}

